\title{
2.
}

\section{Théorèmes sur les formes cubiques et solution d'une équation du quatrième degré à quatre indéterminées.}

(Par Mr. G. Eisenstein à Berlin.)

Je veux énoncer dans celle note un théorème fort singulier sur les formes cubiques qui établit une liaison très remarquable entre la théorie de ces formes et celle de la multiplication des formes quadratiques.

Je nomme forme cubique toute expression telle que

$$
\text { 1. } \quad a x^{3}+3 b x^{2} y+3 c x y^{2}+d y^{3}
$$

où $a, b, c, d$ sont des nombres entiers donnés et $x, y$ des indéterminées. En formant l'ensemble de toutes les expressions semblables dans lesquelles cette forme se change par l'application de toutes les substitutions de la forme

$$
\text { 2. } x=\alpha x^{\prime}+\beta y^{\prime \prime}, \quad y=\gamma x^{\prime}+\delta y^{\prime \prime}
$$

les entiers $\alpha, \beta, \gamma, \delta$ étant tels que

$$
\text { 3. } \alpha \delta-\beta \gamma=1 \text {, }
$$

nous aurons ce que je nomme classe de formes cubiques équivalentes, ou classe cubigue.

Chaque forme cubique a pour correspondante une forme quadratique

$$
\text { 4. } \boldsymbol{A} \boldsymbol{x}^{2}+2 \boldsymbol{B} \boldsymbol{x} \boldsymbol{y}+\boldsymbol{C} \boldsymbol{y}^{2}=\boldsymbol{H}=(\boldsymbol{A}, \boldsymbol{B}, \boldsymbol{C})
$$

dont les coëfficients sont liés avec ceux de la forme cubique par les équations très simples

$$
\text { 5. } A=b^{2}-a c, \quad 2 B=b c-a d, \quad C=c^{2}-b d,
$$

et si l'on applique tant à la forme cubique qu'à la forme quadratique une substitution quelconque (2.), la même liaison existera entre les coëfficients des deux nouvelles formes que l'on obtiendra par cette double transformation, ensorte qu'à une classe entière de formes cubiques correspondra toujours une classe complète de formes quadratiques, et que toutes les classes cubiques se distribueront sur diverses clusses quadrutiques: propriété remarquable qui jette un nouveau jour sur la nature des formes cubiques.

Comme ces préliminaires suffiront pour me faire comprendre, voici le thẻorème qui suit. 
${ }_{9}$ Soit $\boldsymbol{D}$ un déterminant quelconque, mais sans diviseur carré. Distinguons parmi les classes du genre principal ceux qui, par leur triplication produisent la classe principale; je dis que pour ces classes il existera toujours une classe cubique qui leur correspondra, et que pour chacune d'elles il n'en existera qu'une seule, tandis qn'aucune classe cubique ne correspondra au reste des classes quadratiques, ni dans le genre principal, ni dans tous les autres genres."

Il en est différemment si le déterminant a un diviseur carré.

La théorie générale de la distribution des classes cubiques sur les classes quadratiques dépend de la considération des classes qui en général prodnisent par leur triplication une classe quadratique quelconque donnée $\boldsymbol{K}$ de l'ordre primitif, mais laquelle peut être produite par la triplication. On pourrait désigner ces classes par le symbole ${ }^{3} / K$; alors si le déterminant est régulier, ce symbole aura une signification réelle et une seule signification pour chaque classe $\boldsymbol{K}$, quand le nombre total des classes (proprement primitives) n'est pas divisible par trois; ; ) mais quand ce nombre est un multiple de trois, il y aura un tiers parmi les classes $\boldsymbol{K}$ pour lequel l'expression ${ }^{3} \boldsymbol{K}$ a une valeur réelle et triple, tandis que pour les autres classes le symbole ${ }^{3} K$ n'a point de signification réelle.

Le théorème précédent est intimément lié avec la proposition suivante.

La lettre $\boldsymbol{D}$ ayant la même signification comme ci-dessus, l'équation indéterminée à quatre variables:

$$
\text { 6. } x_{1}^{2} x_{4}^{2}-3 x_{2}^{2} x_{3}^{2}+4 x_{1} x_{3}^{3}+4 x_{4} x_{2}^{3}-6 x_{1} x_{2} x_{3} x_{4}=4 D
$$

a la propriété, qu'on peut déduire par une formule générale une infinité de solutions d'une seule formule que l'on suppose connue. En effet, soit donnée une solution de cette équation par le système:

on aura généralement

$$
\text { 7. } \xi_{1}, \xi_{2}, \xi_{3}, \xi_{4},
$$

$$
\text { 8. }\left\{\begin{array}{l}
x_{1}=\alpha^{3} \xi+3 \alpha^{2} \gamma \xi_{2}+3 \alpha \gamma^{2} \xi_{3}+\gamma^{3} \xi_{4}, \\
x_{2}=\alpha^{2} \beta \xi_{1}+\left(\alpha^{2} \delta+2 \alpha \beta \gamma\right) \xi_{2}+\left(2 \alpha \gamma \delta+\beta \gamma^{2}\right) \xi_{3}+\gamma^{2} \delta \xi_{4}, \\
x_{3}=\alpha \beta^{2} \xi_{1}+\left(\beta^{2} \gamma+2 \alpha \beta \delta\right) \xi_{2}+\left(2 \beta \gamma \delta+\alpha \delta^{2}\right) \xi_{3}+\gamma \delta^{2} \xi_{4}, \\
x_{4}=\beta^{3} \xi_{1}+3 \beta^{2} \delta \xi_{2}+3 \beta \delta^{2} \xi_{3}+\delta^{3} \xi_{4},
\end{array}\right.
$$

* Mr. Lejeune Dirichlet a désigné le premier ce nombre remarquable par un procédé fort ingénieux. Voyez, Recherches sur diverses applications etc. Vol. 19 et 21 de ce journal." 
les entiers $\alpha, \beta, \gamma$ étant tels que

$$
\text { 9. } \alpha \delta-\beta \gamma=1 \text {. }
$$

De cette manière la totalité des solutions de l'équation proposée pourra tonjours être distribuée dans un nombre de groupes distincts, en comprenant dans un même groupe deux solutions qui se déduisent l'une de l'autre par les équations (8.). Le nombre de ces groupes est exactement celui qui désigne le nombre des classes quadratiques pour le déterminant $\boldsymbol{D}$ qui, par leur triplication produisent la classe principale, nombre qui pour un déterminant régulier est ou trois ou l'unité, selon que le nombre total des classes est ou n'est pas divisible par trois, mais qui peut être assez considérable pour un déterminant irrégulier.

Voici encore un autre théorème sur expression des nombres par des formes cubiques que j'ai tiré de la même source, et qui n'est pas moins élégant. , , Soit

10. $a x^{3}+3 b x^{2} y+3 c x y^{2}+d y^{3}=f$

une forme cubique donnée, pour laquelle $b c$ - $a d$ est un nombre pair; soit de plus

$$
b^{2}-a c=A, \quad b c-a d=2 B, \quad c^{2}-b d=C, \quad B^{2}-A C=D .
$$

Cela posé, si nous désignons par

$$
\text { 11. } \mathbf{N}, \mathbf{N}^{\prime}, \mathbf{N}^{\prime \prime}, \mathbf{N}^{\prime \prime \prime} \text {, etc. }
$$

la série de tous les nombres différents et premiers avec $2 \boldsymbol{D}$ qui peuvent être représentés par la forme quadratique

$$
\text { 12. }(\boldsymbol{A}, \boldsymbol{B}, \boldsymbol{C})=\boldsymbol{A} \boldsymbol{x}^{2}+2 \boldsymbol{B} x \boldsymbol{y}+\boldsymbol{C} y^{2},
$$

de manière que les valeurs des indéterminées soient premières entre elles: je dis que tous les nombres premiers à $2 \boldsymbol{D}$, exprimables par la forme cubique $f$ et par des valeurs des indéterminées premières entre elles, seront donnés par les valeurs de $\boldsymbol{V}$ qui se trouvent parmi les solutions des équations suivantes:

13. $U^{2}-D V^{2}=N^{3}, \quad U^{2}-D V^{2}=N^{\prime 3}$, etc. etc.

$\boldsymbol{U}$ et $\boldsymbol{V}$ étant des nombres premiers entre eux."

On verra par cet abrégé, que la nouvelle théorie, dont je viens de donner quelques résultats est succeptible à beaucoup de développements, et quelle se recommande à l'attention des géomètres.

Peut être communiquerai je plus tard la théorie complète que j'ai formée de cette partie tout-à-fait nouvelle de la théorie des nombres. 
Je profite de cette occasion pour donner quelques formules nouvelles sur les fonctions elliptiques, mais qui ne sont que des cas bien particuliers d'une équation très-générale. Les voici. On a

$$
\begin{aligned}
& 1+\frac{x}{R}+\frac{x^{2}}{R^{4}}+\frac{x^{3}}{R^{2}}+\ldots .+\frac{x^{n}}{R^{n^{2}}}+\text { in inf. } \\
& =\frac{1}{1-\frac{x}{R-\left(1-R^{2}\right) x}} \\
& \boldsymbol{R}^{2}-\frac{x}{R^{3}-\frac{\left(1-R^{4}\right) x}{x}} \\
& R^{4}-\frac{x}{R^{5}-\frac{\left(1-R^{6}\right)}{R^{6}-\text { etc. in inf. }}}
\end{aligned}
$$

Soit $m$ un nombre impair et $\varrho$ une racine primitive de l'équation

$$
\mathbf{Z}^{m}=1
$$

on aura

$$
\begin{aligned}
& 1+\varrho x+\rho^{4} x^{2}+\rho^{9} x^{3}+\ldots+\rho^{(m-1)^{2}} x^{m-1} \\
& =\frac{1-x^{m}}{1-\frac{x}{\rho}} \quad \text { fraction continue et finie. } \\
& \varrho^{m-1}-\frac{\left(1-\varrho^{m-2}\right) x}{\rho^{m-2}-\operatorname{etc} .} \\
& \rho^{2}-\frac{(1-\rho) x}{\rho-\frac{x}{1-\frac{\left(1-\varrho^{m-1}\right) x}{\rho^{m-1}-\operatorname{etc} .}}} \\
& \rho^{3}-\frac{\left(1-\rho^{2}\right) x}{\rho^{2}-\frac{x}{\rho}}
\end{aligned}
$$

En posant

$$
\begin{gathered}
K=\int_{0}^{\frac{b}{\pi} \pi} \frac{d \phi}{\sqrt{\left(1-k^{2} \sin ^{2} \varphi\right)}}, \quad K^{\prime}=\int_{0}^{\frac{1}{2} \pi} \frac{d \varphi}{\sqrt{\left(1-k^{2} \sin ^{2} \varphi\right)}} \\
k^{2}+k^{\prime 2}=1, \quad p=e^{\pi \frac{K^{\prime}}{K}}
\end{gathered}
$$

on aura 
3. Eisenstein, sur les formes cubiques etc.

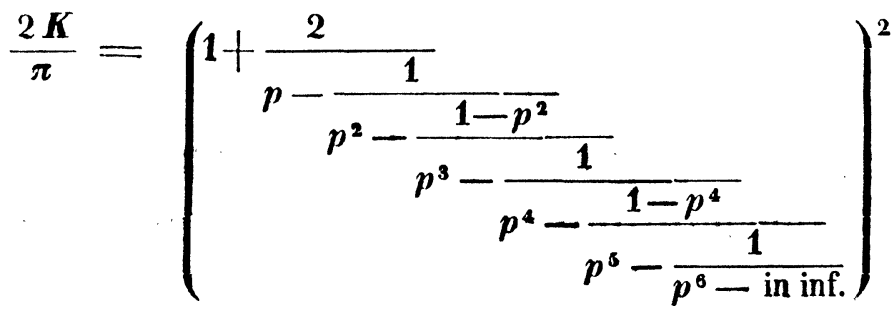

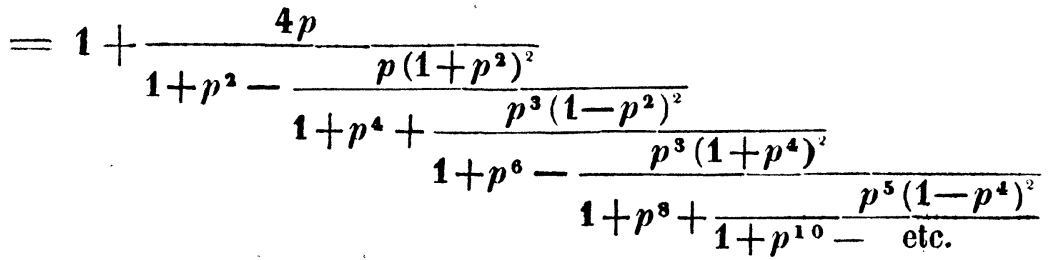

Je n'entre pas dans d'autres détails sur ces résultats, en espérant que j'aurai l'occasion d'offrir aux géomètres la théorie entière assez étendue qui alors contiendra leur démonstration.

Berlin en Décembre 1843. 